\title{
miR-342 inhibits glioma cell proliferation by targeting GPRC5A
}

\author{
JIANJIAO WANG $^{1 *}$, YAN YANG ${ }^{2 *}$, YUANDONG CAO $^{2}$ and XINYU TANG ${ }^{2}$ \\ ${ }^{1}$ Department of Neurosurgery, The Second Affiliated Hospital of Harbin Medical University, Harbin, Heilongjiang 150086; \\ ${ }^{2}$ Department of Radiation Oncology, The First Affiliated Hospital of Nanjing Medical University, \\ Nanjing, Jiangsu 210029, P.R. China
}

Received November 2, 2018; Accepted April 9, 2019

DOI: $10.3892 / \mathrm{mmr} .2019 .10242$

\begin{abstract}
Accumulating evidence suggests that microRNAs (miRNAs) play a key role in the biological behaviors and progression of glioma. However, the function and bio-molecular mechanisms of miR-342 in glioma remain largely unclear. In the present study, reverse transcription quantitative-polymerase chain reaction and western blotting were performed to determine the mRNA and protein expression levels of the factors investigated. MTT assay was performed to examine the proliferation rates. Luciferase reporter assay was performed to test the binding between miRNA-342 and its putative target. Data indicated that miR-342 expression was markedly decreased in human glioma tissues and cell line U87, and reduced miR-342 expression significantly promoted cell proliferation. In order to explore the mechanisms, G-protein coupled receptor family C group 5 member A (GPRC5A) was identified as a target of miR-342 and depletion of GPRC5A suppressed cell proliferation. Our findings demonstrated that miR-342 regulates the cell proliferation of glioma by targeting GPRC5A, which indicates that miR-342 is a target of interest regarding the treatment of refractory glioma, and it may provide a promising prognostic and therapeutic strategy for glioma treatment.
\end{abstract}

\section{Introduction}

Gliomas are the most common malignant tumors of the central nervous system, accounting for approximately $40 \%$ of all brain tumors (1). Despite recent advances including adjuvant chemotherapy, radiotherapy and extensive tumor resection,

Correspondence to: Professor Yuandong Cao or Professor Xinyu Tang, Department of Radiation Oncology, The First Affiliated Hospital of Nanjing Medical University, 300 Guangzhou Road, Nanjing, Jiangsu 210029, P.R. China

E-mail: caoyuandong@jsph.org.cn

E-mail: tangxiny_8379@qq.com

${ }^{*}$ Contributed equally

Key words: GPRC5A, cell proliferation, miR-342, glioma the prognosis of glioma patients and the 5-year survival rate remain unfavorable (2). Thus, there is an urgent need to identify the mechanisms involved in glioma progression and metastasis to elucidate novel diagnostic and therapeutic targets for this malignancy.

MicroRNAs (miRNAs), single-stranded RNAs with a length of approximately 19-24 nucleotides, play significant roles in a series of biological cellular processes such as cell proliferation, migration, invasion and tumorigenesis (3). miR-342 serves a critical role in numerous physiological and pathological processes. It has been reported to be involved in the pathogenesis of many types of cancers, such as gastric cancer (4), hepatocellular carcinoma (5) and non-small cell lung cancer (6). Although these studies have demonstrated the important role of miR-342 in cancer progression, the modes of action of miR-342 in glioma have not been fully characterized.

G-protein-coupled receptors are the largest protein superfamily with more than 700 genes in the human genome (7). They play an important role in a variety of biological processes (8). It has been demonstrated that GPRC5A, one member of the GPCR family, is upregulated in many cancer tissues and cell lines (9-11). Yet, the relationship between GPRC5A and miR-342 remains unclear. We hypothesized that miR-342 directly targets GPRC5A, and the present study was designed to explore this hypothesis.

In the present study, the expression level of miR-342 was measured to assist the investigation of its regulatory roles in glioma. miR-342 was found to be markedly downregulated in glioma tissues and cell lines, and to exert tumor-suppressing functions in glioma. Moreover, miR-342 was found to regulate cell proliferation by targeting GPRC5A.

\section{Materials and methods}

Clinical samples and cell culture. Human glioma cell lines U-87MG (ATCC HTB-14 (RRID: CVCL_0022, glioblastoma of unknown origin), U251 (The Cell Bank of Type Culture Collection of Chinese Academy of Sciences; cat. no. TCHu 58), T98G [American Type Culture Collection (ATCC); cat. no. CRL-1690] and SNB19 (ATCC; cat. no. CRL-2219) and normal human astrocytes (NHAs; ScienCell Research Laboratories, Inc.; cat. no. 1820) were cultured in Dulbecco's modified Eagle's medium (DMEM; 
$\mathrm{Gibco}^{\mathrm{TM}}$, 10564011) supplemented with $10 \%$ fetal bovine serum (FBS, Gibco ${ }^{\mathrm{TM}}$, 10099141) (both from Thermo Fisher Scientific, Inc., Waltham, MA, USA), $100 \mathrm{IU} / \mathrm{ml}$ penicillin, and $100 \mu \mathrm{g} / \mathrm{ml}$ streptomycin at $37^{\circ} \mathrm{C}$ under a $5 \% \mathrm{CO}_{2}$ atmosphere. Glioma specimens were collected from 39 patients following prior approval and written informed consent. Ten normal brain tissue samples used as controls were collected by donations from individuals who died in traffic accidents. All clinical samples were collected and histologically examined by pathologists from July 2016 to May 2018 at The Second Affiliated Hospital of Harbin Medical University. The present study was approved by The Ethics Committee of The Second Affiliated Hospital of Harbin Medical University. Written informed consent was obtained from all enrolled subjects.

Cell transfection. The miR-342 mimics, inhibitor and their corresponding miRNA negative control (miR-NC) were chemically synthesized by GenePharma (Shanghai, China) and transfected using Lipofectamine 2000 (Invitrogen; Thermo Fisher Scientific, Inc.). Overexpression or knockdown plasmids were transfected using Lipofectamine 2000 following the manufacturer's instructions.

GPRC5A overexpression and shRNA-mediated knockdown plasmids. The longest transcript human genes of GPRC5A (NCBI Reference Sequence: NM_003979.3) were cloned into pcDNA 3.1 plasmids and then sequenced for validation. The siRNA duplexes targeting GPRC5A were obtained from Invitrogen; Thermo Fisher Scientific, Inc. In order to knock down GPRC5A expression in U87 cells, subconfluently cultured U87 cells were transfected with GPRC5A shRNA, or negative control shRNA using the RNAiMAX transfection reagent (Invitrogen; Thermo Fisher Scientific, Inc.), according to the manufacturer's protocol. GPRC5A expression was assessed following 3 days. The shRNAs were designed by Invitrogen (Invitrogen; Thermo Fisher Scientific, Inc.) and cloned using Lipofectamine 2000 (Invitrogen; Thermo Fisher Scientific, Inc.), according to the manufacturer's protocol. Stable population transfection was obtained following selection with $1 \mu \mathrm{g} / \mathrm{ml} \mathrm{G} 418$ (Amresco, LLC, Solon, OH, USA) for 2 weeks. All shRNA oligos were obtained from Invitrogen; Thermo Fisher Scientific, Inc.

Quantitative real-time PCR ( $P P C R)$. Trizol reagent (Invitrogen; Thermo Fisher Scientific, Inc.) was used for total RNA extraction. Six microliters of the extracted RNA was reverse transcribed using the PrimeScript ${ }^{\mathrm{TM}}$ RT reagent kit with gDNA Eraser (Takara Bio, Inc., Otsu, Japan) according to the provider's protocol. Quantitative PCR was performed using SYBR ${ }^{\circledR}$ Green Real-Time PCR Master Mix (Takara) in the StepOnePlus Real-Time System (Applied Biosystems ${ }^{\mathrm{TM}}$ ABI Prism 7500 Fast; Thermo Fisher Scientific, Inc.). The sequences of primers used were: GPRC5A forward 5'-CGC CACAAAGCAACGAA-3' and reverse primer 5'-ATAGAG CGTGTCCCCTGTCT-3'; GAPDH forward 5'-GAAAGC CTGCCGGTGACTAA-3' and reverse primer 5'-GCATCA CCCGGAGGAGAAAT-3'; U6 small nuclear RNA forward 5'-CTCGCTTCGGCGCACA-3' and reverse primer: 5'-AAC GCTTCACGAATTTGCGT-3'; miR-342 forward 5'-AGGTGA
GGGGTGCTATCTGT-3' and reverse primer 5'-GGGTGC GATTTCTGTGTGAG-3'. All the samples were amplified in triplicate and each experiment was repeated three times. The conditions for the real-time fluorescent quantitative PCR were: 1 cycle at $95^{\circ} \mathrm{C}$ for $5 \mathrm{~min}$ in the holding stage; 40 cycles at $95^{\circ} \mathrm{C}$ for $15 \mathrm{sec}$ and $60^{\circ} \mathrm{C}$ for $60 \mathrm{sec}$ in the cycling stage; 1 cycle at $95^{\circ} \mathrm{C}$ for $15 \mathrm{sec}, 60^{\circ} \mathrm{C}$ for $1 \mathrm{~min}$ and $95^{\circ} \mathrm{C}$ for $15 \mathrm{sec}$ in the melt curve stage. Thermal cycling and real-time detection were conducted using the StepOnePlus Real-Time PCR Systems (ABI, Thermo Fisher Scientific, Inc.). The quantities of each mRNA were calculated using the comparative $\left(2^{-\Delta \Delta \mathrm{Cq}}\right)$ method (12).

Western blot analysis. Protein was isolated from the cells and tissues with RIPA lysis buffer containing $1 \%$ protease inhibitor cocktails (Pierce Biotechnology, Inc.; Thermo Fisher Scientific, Inc.). After sample buffer was added to the proteins (each well, $30 \mu \mathrm{g}$ per sample), proteins were boiled at $95^{\circ} \mathrm{C}$ for $10 \mathrm{~min}$. Then, the proteins were separated using $10 \%$ polyacrylamide gel electrophoresis. After electrophoresis, proteins were transferred to polyvinylidene fluoride (PVDF) membranes with $100 \mathrm{~V}$ transfer-molded voltage lasting for 45 to $70 \mathrm{~min}$. After determination of the protein concentration, primary antibodies for western blotting were applied which included anti-GPRC5A (dilution, 1:1,000; PAB14597; Abnova, Taipei, Taiwan) and anti-GAPDH (dilution, 1:2,000; ab8245; Abcam, Cambridge, UK). HRP-conjugated IgG $(1: 5,000)$ antibody was used as the secondary antibody. After which membranes were washed 3 times ( $5 \mathrm{~min} /$ time). Development was completed with chemiluminescence reagents. GAPDH was used as an internal reference. Bands were visualized with a Bio-Rad Gel Doc EZ imager (Bio-Rad Laboratories, Inc., Hercules, CA, USA). The specific bands were visualized with a chemiluminescence system (Millipore), and then visualized with Quantity One software 4.6.2 (Bio-Rad Laboratories, Inc.).

Cell Counting Kit-8 (CCK-8) assay. Cells in the logarithmic growth phase were digested with trypsin and seeded on 96-well plates at $100 \mu \mathrm{l}$ of medium containing $1 \times 10^{4}$ cells per well. Then we measured the cell proliferation rate at $0,24,48$, and $72 \mathrm{~h}$ after transfection. Ten microliters of CCK-8 reagent was added into each well following another 2-h incubation at $37^{\circ} \mathrm{C}$. The absorbance value was determined by using the XT-96DJ ELISA analyzer at a wavelength of $490 \mathrm{~nm}$.

Luciferase reporter assay. Wild-type and mutated GPRC5A 3'-UTR containing the putative binding site of miR-342 were synthesized and sequenced. Cells were seeded in 24-well plates and transfected with reporter vectors together with miR-342 mimics, miR-342 inhibitor or the corresponding miR-NC. The firefly luciferase activity was measured and normalized to Renilla signals at $48 \mathrm{~h}$ post-transfection.

Tumorigenicity assay. In total, $16 \mathrm{BALB} / \mathrm{c}$ male nude mice (specific pathogen-free grade; weight, 16-18 g; age, 4-6 weeks) were purchased from the Laboratory Animal Center of Harbin Medical University. Lentiviral-mediated stable GAPC5A, GAPC5A+miR-342, miR-342 mimic cells and miR-NC cells were resuspended in Hank's buffer and mixed with an equal 
volume of Matrigel (BD Biosciences) at a concentration of $5 \times 10^{6}$ cells $/ \mathrm{ml}$. The cells were subcutaneously injected into the flanks of nude mice ( $\mathrm{n}=4$ in each group). Subsequently, the mice were maintained in a specific pathogen-free grade laboratory, under the following conditions: Controlled temperature, $23 \pm 2^{\circ} \mathrm{C}$; humidity, $40-70 \%$; 12 -h light/dark cycle) at the Laboratory Animal Center in our hospital with ad libitum access to food and water for 4 weeks. The volume of xenograft tumors was monitored every 3 days by measuring the length and width (Volume=length $\mathrm{x}$ width $\mathrm{x}$ width/2). The animal study was conducted in accordance with the Institutional Animal Care and Use Committee (IACUC) guidelines, and was approved by the Experimental Animal Ethics Committee of The Second Affiliated Hospital of Harbin Medical University.

Statistical analysis. All values are expressed as mean \pm SEM and were analyzed by one-way analysis of variance (ANOVA) followed by Tukey's post hoc test among groups using Statistical Product and Service Solutions (SPSS) (version 17.0) (SPSS, Inc., Chicago, IL, USA). Pearson's correlation analysis was performed to study the correlation between the expression of miR-342 and GPRC5A in cancer tissues. A P-value $<0.05$ was considered to indicate a statistically significant difference between groups.

\section{Results}

miR-342 is downregulated and GPRC5A is upregulated in glioma tissues and cell lines. Downregulation of miR-342 was observed in the glioma tissues $(\mathrm{P}<0.01$; Fig. 1A) and cell lines $(\mathrm{P}<0.05$; Fig. 1B) when compared with that noted in the normal human prostate tissues and the normal human astrocytes (NHAs). Meanwhile, the western blot results showed that the protein expression of GPRC5A was significantly upregulated in human glioma tissues (Fig. 1C) and cell lines (Fig. 1D). According to the results of RT-qPCR, GPRC5A expression in the U87 cell line was the highest, therefore, we chose this cell line for further experiments. The correlation analysis confirmed that the expression of miR-342 and GPRC5A was significantly negatively correlated $(r=-0.4559$, $\mathrm{P}=0.0035$; Fig. 1E).

miR-342 inhibits the proliferation of glioma cells. Next, we explored the potential role of miR-342 in glioma. The transfection efficiency was determined according to the level of miR-342 using RT-qPCR. As shown in Fig. 2A, a significantly increased expression of miR-342 was achieved after miR-342 mimic transfection and a significantly decreased expression of miR-342 was achieved after miR-342 inhibitor transfection. Moreover, upregulation of miR-342 resulted in greater suppression of cell proliferation than the control (mimics NC), whereas downregulation of miR-342 promoted cell proliferation (Fig. 2B) as determined using the CCK-8 assay. These results indicated that miR-342 inhibited the proliferation of U87 and U251 cells and downregulation of miR-342 promoted the proliferation of cells.

GPRC5A is a direct target of miR-342 in glioma. In order to determine the mechanism of miR-342 in cell proliferation, G-protein coupled receptor family $\mathrm{C}$ group 5 member A (GPRC5A) was found to be a putative target of miR-342 (Fig. 3A). At the protein and mRNA levels, overexpression or knockdown of miR-342 resulted in a significant decrease or increase in the expression level of GPRC5A, respectively (Fig. 3B and C). In addition, luciferase reporter assays were performed to ascertain whether miR-342 targets GPRC5A by binding to its 3'UTR. The results from the luciferase reporter assay indicated that upregulated expression of miR-342 significantly inhibited the activity of the reporter gene, whereas miR-342 inhibitor significantly increased it (Fig. 3D). The results indicate that GPRC5A is a direct target of miR-342.

GPRC5A promotes cell proliferation of U87. In order to investigate the cellular function of GPRC5A, the expression level of GPRC5A in U87 cells was manipulated by transfection with an overexpression (OE) or shRNA-mediated knockdown plasmids. The mRNA and protein levels of GPRC5A were determined in the transfected cells for which the expression levels of GPRC5A were markedly increased in the presence of overexpression plasmids or decreased in the absence of plasmids or silenced by shRNA. The inhibitory effect of each shRNA (sh1, sh2, sh3) was not significantly different while sh2 had the highest inhibition rate of GPRC5A, thus this shRNA was selected for further experiments (Fig. 4A and B). Moreover, CCK-8 assay results showed that overexpression of GPRC5A significantly promoted cell growth, while knockdown of GPRC5A suppressed it (Fig. 4C). The observation indicated GPRC5A expression associated with cell proliferation in vitro.

Restoration of GPRC5A reverses the effects of $m i R$-342 in vitro and in vivo. Based on the above results, it was proposed that miR-342 suppresses cell proliferation via up-regulation of GPRC5A. Considering the low expression level of miR-342, rescue experiments were performed by co-transfecting the miR-342 mimics with or without GPRC5A followed by determination of the cell proliferation of U87 cells. Growth curves obtained by CCK- 8 assay showed that upregulation of the expression of miR-342 alone significantly inhibited cell growth whereas overexpression of GPRC5A alone significantly promoted cell growth; co-expression of GPRC5A with miR-342 abrogated the inhibitory effects of miR-342 mimics on the cell proliferation (Fig. 5A). Based on the in vitro data, the effect of miR-342 and GPRC5A on tumor growth was further evaluated in vivo. Lentivirus of miR-342 and GPRC5A were either used to infect cells alone or simultaneously. We found that tumor xenografts derived from cells infected with miR-342 alone were significantly smaller than the negative control while those infected with GPRC5A showed an opposite trend; co-infection of GPRC5A with miR-342 abrogated the inhibitory effects of miR-342 (Fig. 5B). The expression level of GPRC5A in tumor xenografts was also assessed. The expression level of GPRC5A was much lower in the miR-342 mimic group and higher in the GPRC5A overexpression or co-infected groups, compared with their counterparts in the negative control (NC) group (Fig. 5C). The in vitro and in vivo observations suggest that miR-342 targets the $3^{\prime} \mathrm{UTR}$ of GPRC5A directly and inhibits U87 cell proliferation via GPRC5A up-regulation. 
A

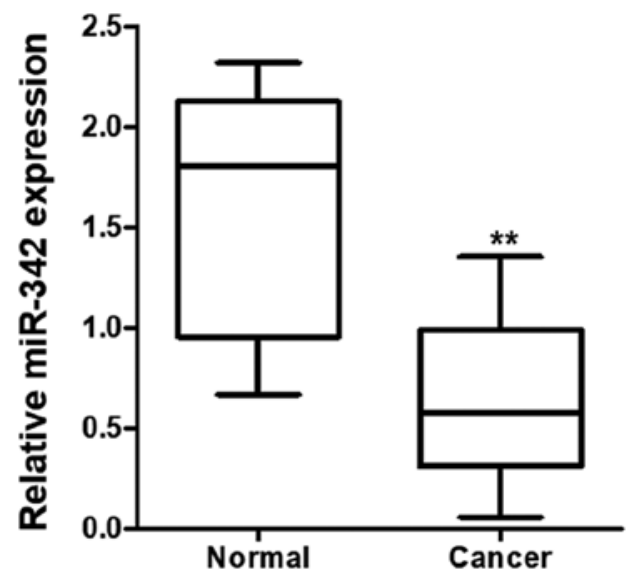

C

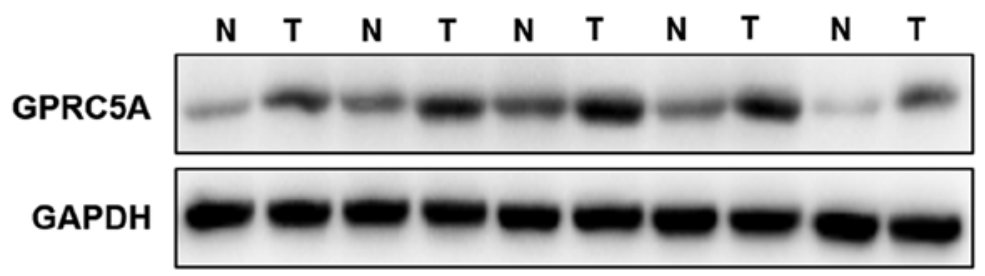

B

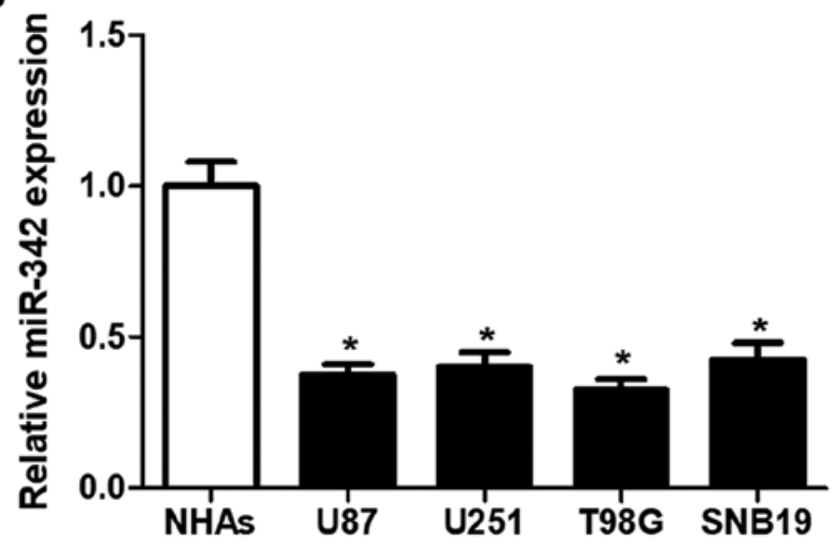

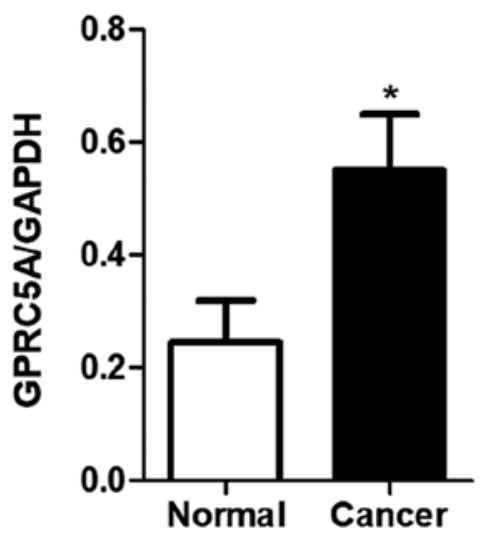

D
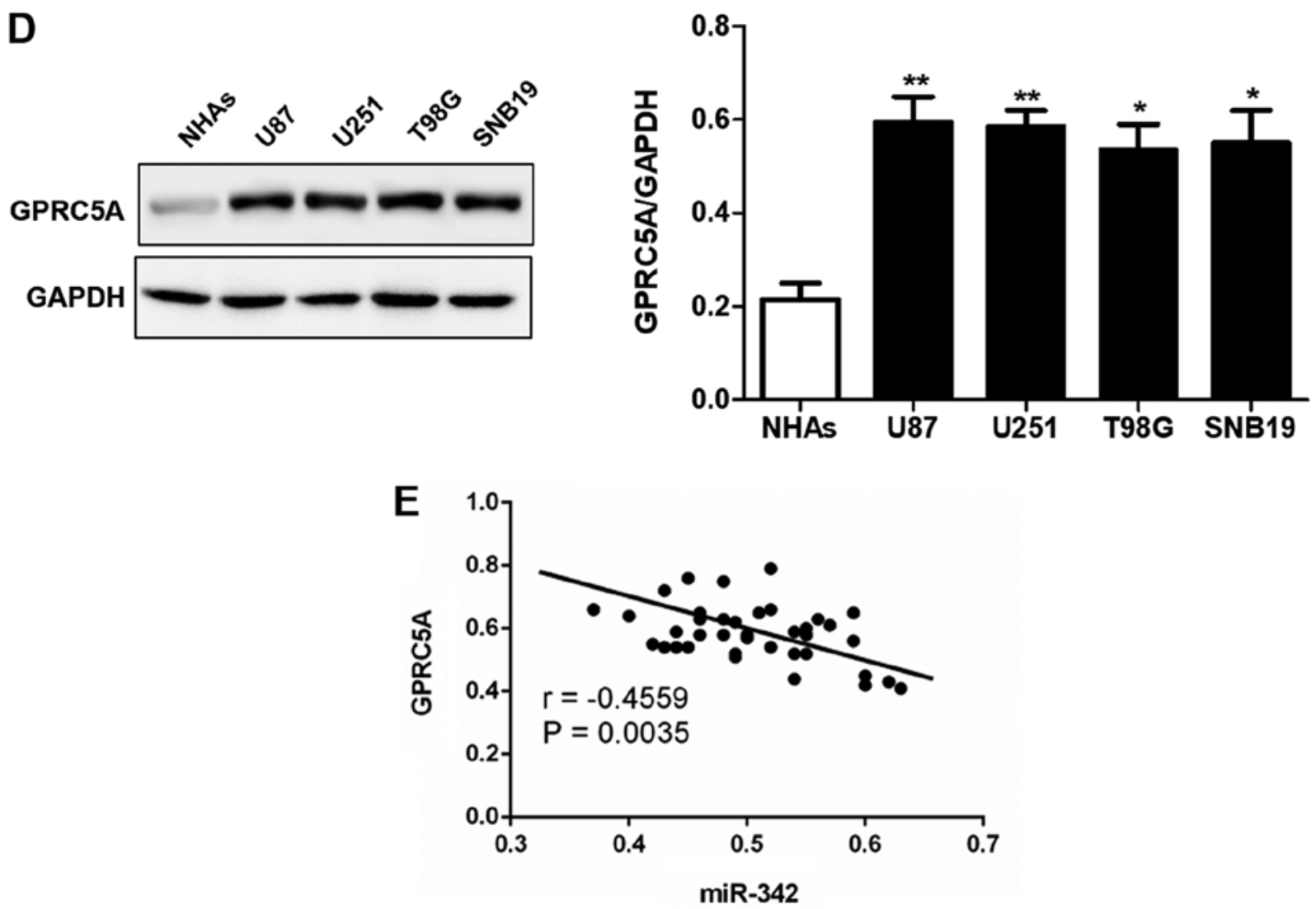

Figure 1. miR-342 is downregulated in human glioma cancer tissues and glioma cell lines. (A) Expression of miR-342 in human glioma cancer tissues and adjacent non-tumor tissues were detected by quantitative real-time PCR. (B) Expression of miR-342 in several glioma cell lines and normal human astrocytes (NHAs) was detected by quantitative real-time PCR. (C) Protein levels of GPRC5A in human glioma cancer tissues and adjacent non-tumor tissues were detected by western blot analysis. T, tumor tissue; N, normal tissue. (D) Protein levels of GPRC5A in several glioma cell lines and normal human astrocytes (NHAs) were detected by western blot analysis. (E) Correlation analysis between expression of miR-342 and GPRC5A in cancer tissues. ${ }^{*} \mathrm{P}<0.05,{ }^{* *} \mathrm{P}<0.01$, compared to normal non-tumor tissues or NHAs. GPRC5A, G-protein coupled receptor family C group 5 member A. 

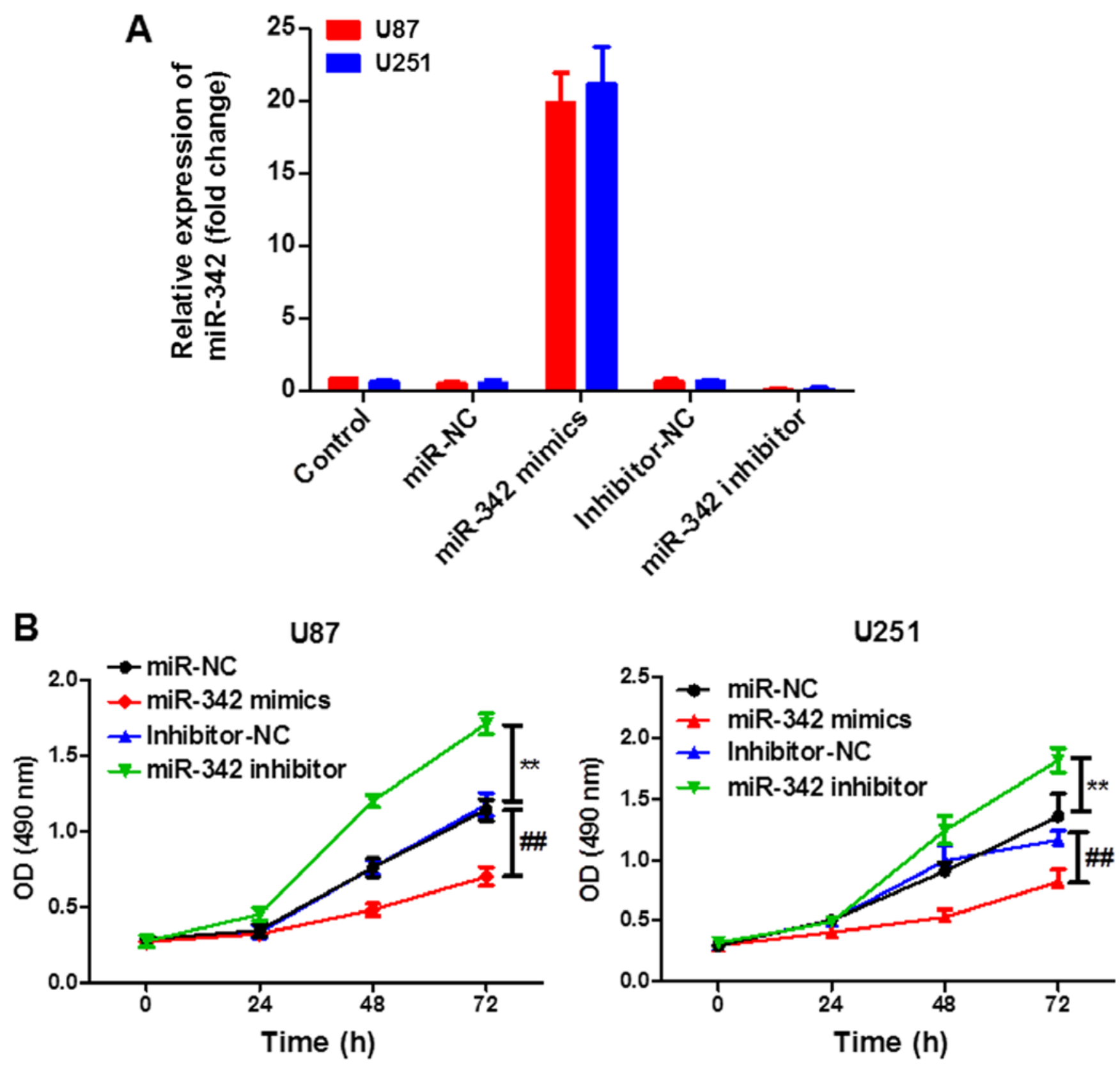

Figure 2. Overexpression of miR-342 inhibits glioma cancer cell proliferation in vitro. (A) The expression of miR-342 in the U87 and U251 cell lines following transfection with miR-NC, miR-342 mimics, inhibitor-NC or miR-342 inhibitor as detected by quantitative real-time PCR. (B) The proliferation ability of the glioma U87 and U251 cell lines following transfection with miR-NC, miR-342 mimics, inhibitor-NC or miR-342 inhibitor, as measured by CCK-8 assay. ${ }^{* *} \mathrm{P}<0.01$ vs. miR-NC; ${ }^{\#} \mathrm{P}<0.01$ vs. Inhibitor-NC. GPRC5A, G-protein coupled receptor family C group 5 member A.

\section{Discussion}

Gliomas are the most common primary brain tumors, which show an extremely high proliferation and invasive capacity $(13,14)$. Therefore, effective diagnostic and therapeutic strategies for glioma are urgently needed. Recently, studies have confirmed that miRNAs play important roles in tumorigenesis and development, and are involved in the regulation of tumor growth, apoptosis, differentiation, invasion, angiogenesis, and metastasis $(15,16)$. In addition, miRNAs can also act as an oncogenes or tumor-suppressor genes, and their role in the development of gliomas acts on the mechanisms of glioma (17). Downregulation of miR-342 has been found in a number of cancer types, such as breast cancer (18), prostate cancer (19) and nasopharyngeal carcinoma (20). Thus, it has been proposed that miR-342 could be used as a diagnostic and prognostic biomarker. In the present study, a significantly downregulated miR-342 level was observed in glioma tissues and cell lines and the increase in the expression level of miR-342 was found to suppress the proliferation of glioma cells in vitro, suggesting that miR-342 functions as an anti-oncogene.

G-protein-coupled receptors are the largest protein superfamily with more than 700 genes in the human genome (7) playing an important role in a variety of biological processes (8). This protein superfamily also acts as drug targets in many different diseases and more than $40 \%$ of FDA (Food and Drug Administration)-approved drugs target 
A
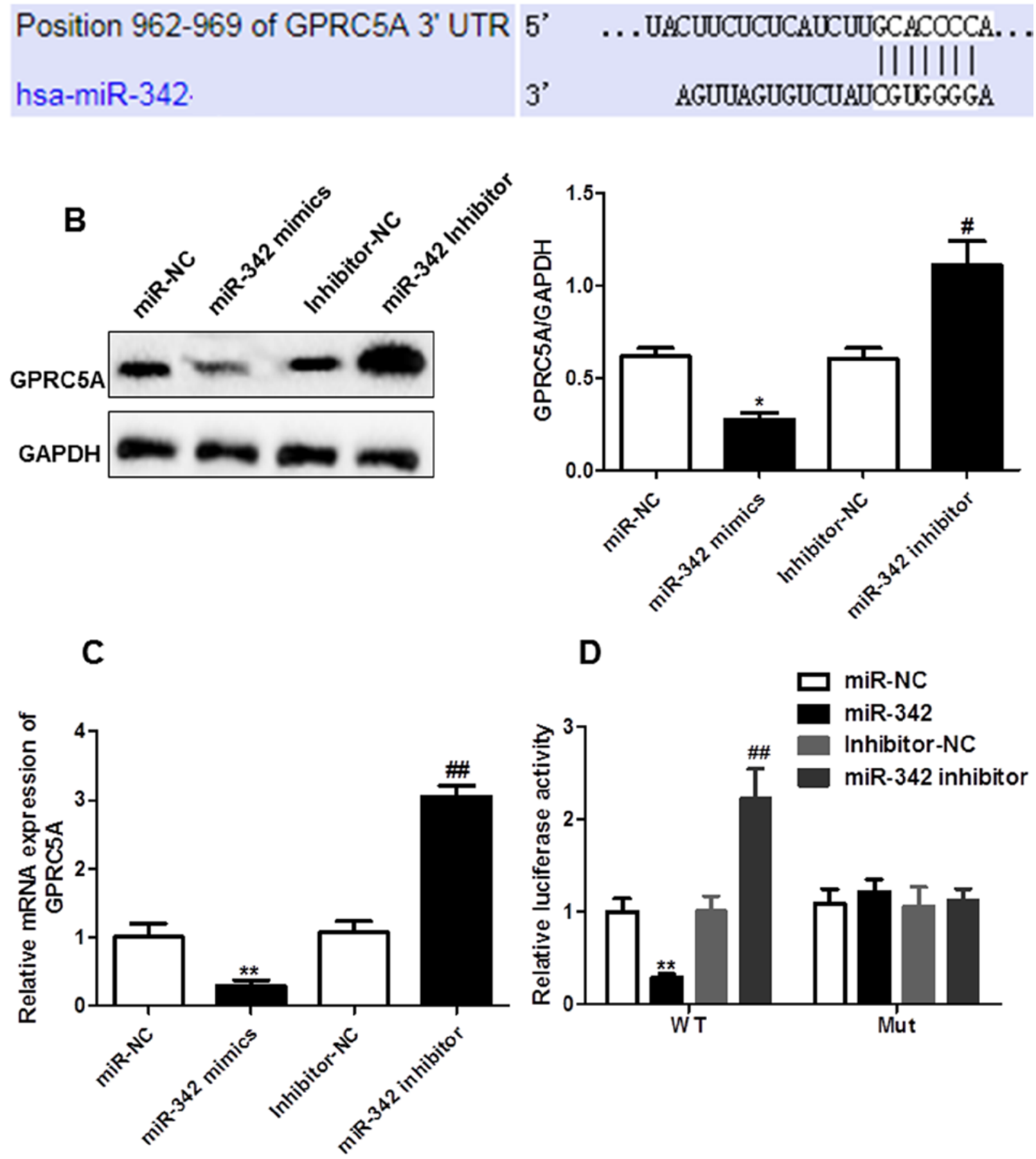

Figure 3. miR-342 directly targets GPRC5A in U87 cells. (A) Sequence complementary pairings of miR-342 with GPRC5A wild-type (WT) and mutant (Mut) 3'UTR are shown. (B) Protein levels of GPRC5A in U87 cells transfection with miR-NC, miR-342 mimics, inhibitor-NC or miR342-inhibitor were determined by western blot analysis. Representative images of western blot were shown, bands were quantitated by densitometry and normalized against GAPDH. (C) mRNA levels of GPRC5A in U87 cells transfected with miR-NC, miR-342 mimics, inhibitor-NC or miR342-inhibitor were determined by reverse transcription-quantitative PCR. (D) Luciferase activities were determined in U87 cells $48 \mathrm{~h}$ after co-transfection with miR-NC, miR-342 mimics, inhibitor-NC or miR342-inhibitor and juciferase reporter vector containing WT or mutants of GPRC5A $3{ }^{\prime} \mathrm{UTR} .{ }^{*} \mathrm{P}<0.05,{ }^{* *} \mathrm{P}<0.01 \mathrm{vs}$. miR-NC; ${ }^{*} \mathrm{P}<0.05$, ${ }^{\# \#} \mathrm{P}<0.01 \mathrm{vs}$. Inhibitor-NC. GPRC5A, G-protein coupled receptor family C group 5 member A.

GPCRs (G protein-coupled receptors) or GPCR-associated pathways (21). GPCRs also play an integral role in regulating and activating cancer-associated signaling pathways; therefore, they may be used as biomarkers for the early diagnosis of various types of cancer (22). Further investigation of the pharmacological potential of GPCRs and their downstream regulators is required in order to develop therapies that can efficiently target cell signaling pathways in cancer $(23,24)$.

GPRC5A, also termed RAI3 (retinoic acid-induced protein 3), located on chromosome 12p13-p12.3, has been found 
A

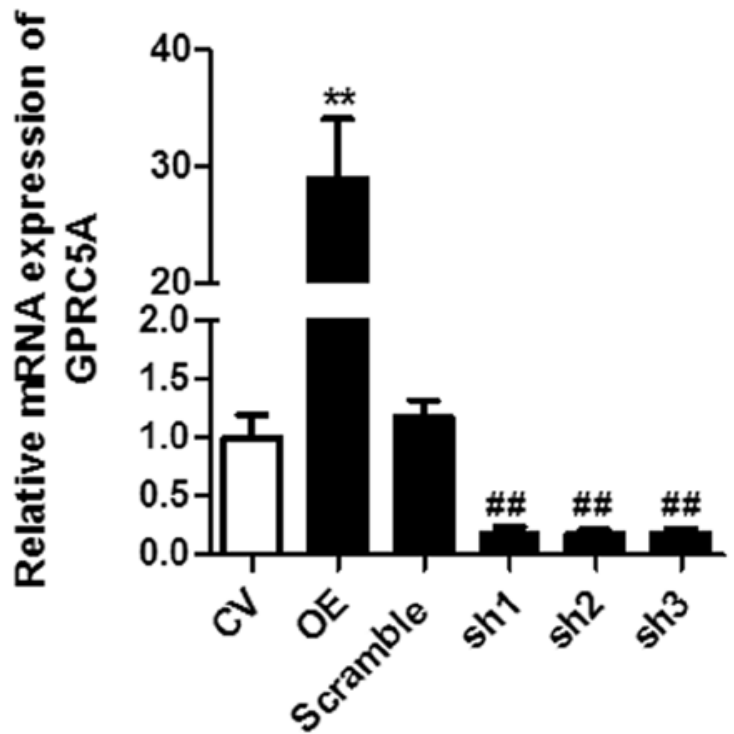

C

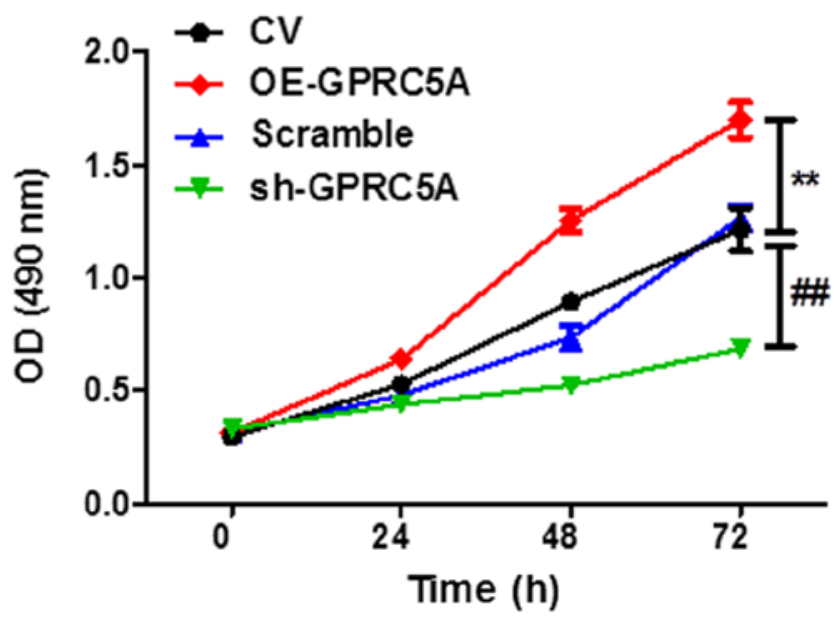

B
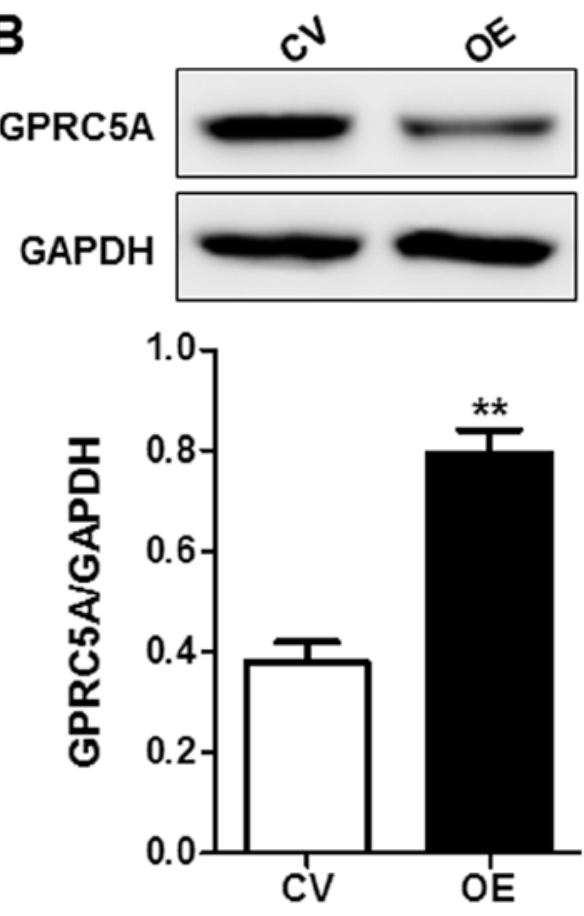
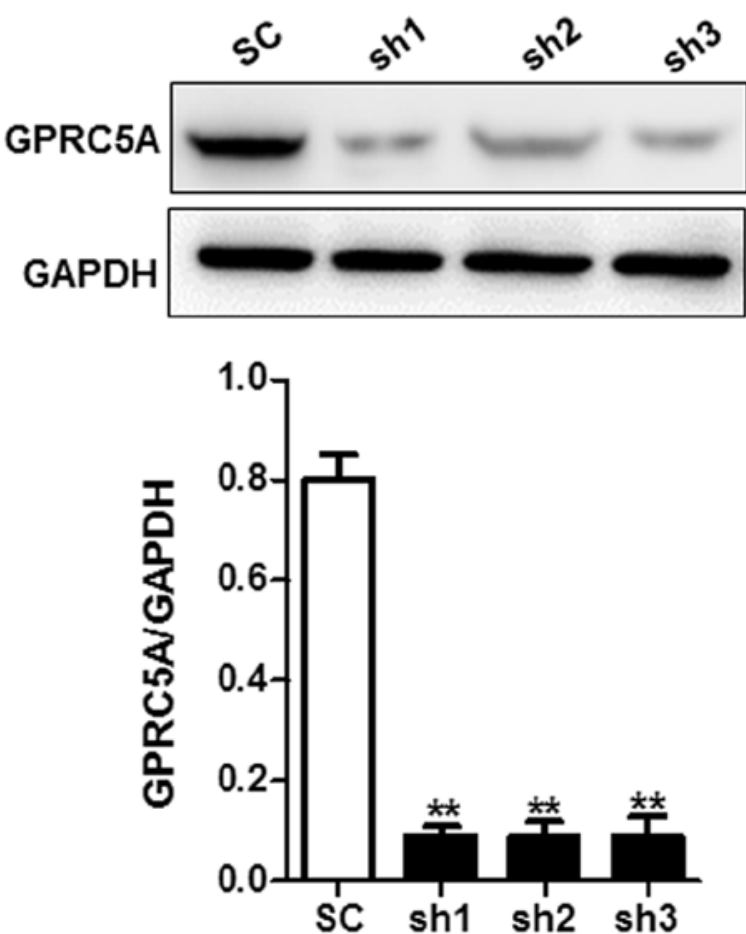

Figure 4. GPRC5A promotes U87 cell proliferation in vitro. (A) mRNA levels of GPRC5A in the cells transfected with the GPRC5A overexpression (OE) plasmids or shRNA-mediated knockdown plasmids were determined by quantitative real-time PCR. (B) Proteins levels were also confirmed by western blot analysis. Representative images of the western blots are shown; bands were quantitated by densitometry and normalized against GAPDH. (C) Growth curves of each group were obtained by CCK-8 assay in U87 cells transfected with OE GPRC5A or shRNA of GPRC5A plasmids. ${ }^{* *} \mathrm{P}<0.01$ vs. CV or SC; ${ }^{\#} \mathrm{P}<0.01$ vs. Scramble. GPRC5A, G-protein coupled receptor family C group 5 member A.

to play significant roles in various biological processes, such as cell proliferation and the cell cycle. However, the influences of GPRC5A on different cancers vary. GPRC5A was reported to be strongly expressed in the lung (25) and is regarded as a tumor suppressor in lung cancer as well as in head and neck squamous cell carcinoma (26). However, there are many studies that have reported that high expression of GPRC5A is correlated with a worse survival rate in colon, breast and gastric cancer (27). Liu and colleagues found high expression of GPRC5A in pancreatic cancer and it was found to suppress the activity of phosphorylated GSK-3 $\beta$ at Ser9 (26). Moreover, Zhou and Rigoutsos reported that miR-103a-3p also targets the 5'UTR of GPRC5A and reduced GPRC5A protein expression in pancreatic cells. It also may indirectly regulate many 

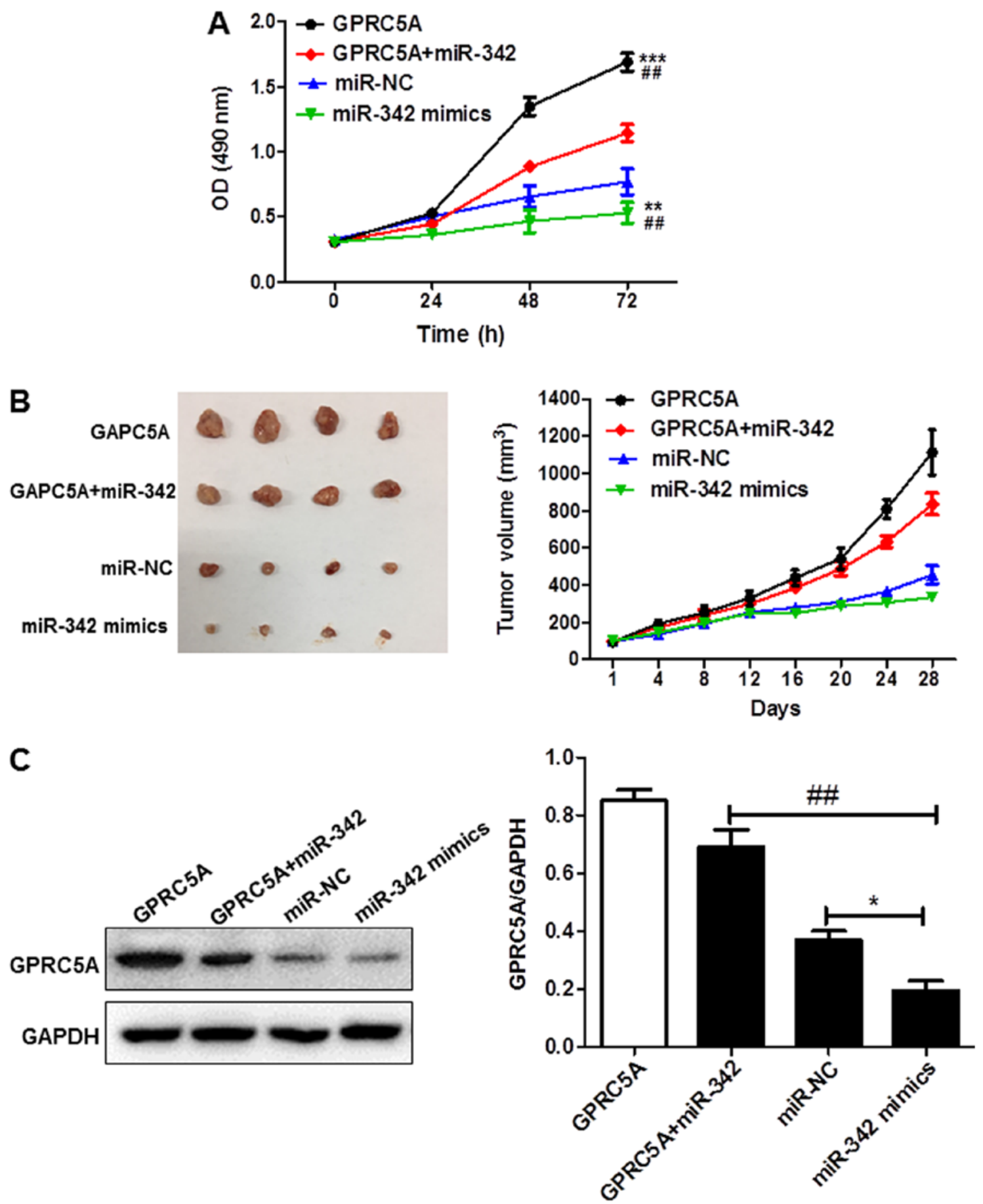

Figure 5. Upregulation of GPRC5A reverses the inhibitory effects of miR-342 mimics on U87 cells. (A) Growth curves were obtained by CCK-8 assay in the cells transfected with miR-342 mimic, GPRC5A overexpression plasmid alone or co-transfected with miR-342 mimic and GPRC5A overexpression plasmid or the negative control (NC). (B) Representative images of tumor xenografts and tumor growth curves are shown. (C) Protein levels of GPRC5A9 in each group were determined by western blot analysis. ${ }^{*} \mathrm{P}<0.05,{ }^{* *} \mathrm{P}<0.01,{ }^{* * *} \mathrm{P}<0.001$ vs. miR-NC; ${ }^{\# \#} \mathrm{P}<0.01$ vs. GPRC5A+miR-342.

cell processes, such as DNA repair, metabolism and the cell cycle (28). In the present study, we confirmed that GPRC5A promoted the proliferation of U87 cells and confirmed that GPRC5A is a direct target of miR-342. Furthermore, the tumor-suppressive effect of miR-342 was reduced by enforced expression of GPRC5A in vivo and in vitro. These results suggest that miR-342 functions as an anti-oncogene via multiple gene targeting, such as on GPRC5A.

In conclusion, the study availed a better understanding of the function of miR-342 and GPRC5A in glioma. We confirmed the downregulated level of miR-342 in glioma and revealed the role of miR-342 in glioma cell proliferation and invasion. Our data indicated the suppressive role of miR-342 in glioma development and miR-342 may serve as a potential therapeutic target in glioma. However, our results were based on one single cell line, U87, and thus additional cell lines are needed to be included in further research. Meanwhile, although the role of miR-342 in cellular invasiveness and cancer progression is clear, its mechanisms remain to be investigated. 


\section{Acknowledgements}

Not applicable.

\section{Funding}

The present study was supported by the 2017 China Postdoctoral Science Foundation's 62nd Batch of Funded Projects (2017M621787).

\section{Availability of data and materials}

The datasets used during the present study are available from the corresponding author upon reasonable request.

\section{Authors' contributions}

JW, YY, YC and XT interpreted and analyzed the data, and drafted the manuscript. JW analyzed the data. XT designed the study. All authors interpreted the results and wrote the manuscript.

\section{Ethics approval and consent to participate}

The animal study was conducted in accordance with the Institutional Animal Care and Use Committee (IACUC) guidelines, and was approved by the Experimental Animal Ethics Committee of The Second Affiliated Hospital of Harbin Medical University. The present study was approved by the Ethics Committee of The Second Affiliated Hospital of Harbin Medical University. Written informed consent was obtained from all enrolled subjects.

\section{Patient consent for publication}

Not applicable.

\section{Competing interests}

The authors state that they have no competing interests.

\section{References}

1. Gu X, Gong H, Shen L and Gu Q: MicroRNA-129-5p inhibits human glioma cell proliferation and induces cell cycle arrest by directly targeting DNMT3A. Am J Transl Res 10: 2834-2847, 2018.

2. Ji ZG, Jiang HT and Zhang PS: FOXK1 promotes cell growth through activating wnt $/ \beta$-catenin pathway and emerges as a novel target of miR-137 in glioma. Am J Transl Res 10: 1784-1792, 2018

3. Gao Y, Lin L, Li T, Yang J and Wei Y: The role of miRNA-223 in cancer: Function, diagnosis and therapy. Gene 616: 1-7, 2017.

4. Wang F, Liang S, Liu X, Han L, Wang J and Du Q: LINC00460 modulates KDM2A to promote cell proliferation and migration by targeting miR-342-3p in gastric cancer. Onco Targets Ther 11 6383-6394, 2018.

5. Liu W, Kang L, Han J, Wang Y, Shen C, Yan Z, Tai Y and Zhao C: miR-342-3p suppresses hepatocellular carcinoma proliferation through inhibition of IGF-1R-mediated Warburg effect. Onco Targets Ther 11: 1643-1653, 2018.

6. Xue X, Fei X, Hou W, Zhang Y, Liu L and Hu R: miR-342-3p suppresses cell proliferation and migration by targeting AGR2 in non-small cell lung cancer. Cancer Lett 412: 170-178, 2018.

7. Venkatakrishnan AJ, Deupi X, Lebon G, Tate CG, Schertler GF and Babu MM: Molecular signatures of G-protein-coupled receptors. Nature 494: 185-194, 2013.
8. Solinski HJ, Gudermann T and Breit A: Pharmacology and signaling of MAS-related G protein-coupled receptors. Pharmacol Rev 66: 570-597, 2014.

9. Jin E, Wang W, Fang M, Wang W, Xie R, Zhou H, Ye J, Xu R and Ma S: Clinical significance of reduced GPRC5A expression in surgically resected non-small cell lung cancer. Oncol Lett 17: 502-507, 2019.

10. Gu C, Zhou N, Wang Z, Li G, Kou Y, Yu S, Feng Y, Chen L, Yang $\mathrm{J}$ and Tian $\mathrm{F}$ : circGprc5a promoted bladder oncogenesis and metastasis through Gprc5a-targeting peptide. Mol Ther Nucleic Acids 13: 633-641, 2018.

11. Klaschik K, Hauke J, Neidhardt G, Tränkle C, Surowy HM, Heilmann-Heimbach S, Rappl G, Mangold E, Arnold N, Niederacher D, et al: The GPRC5A frameshift variant c.183del is not associated with increased breast cancer risk in BRCA1 mutation carriers. Int J Cancer 144: 1761-1763, 2019.

12. Jing L, Li H, Zhang T, Lu J and Zhong L: MicroRNA-4530 suppresses cell proliferation and induces apoptosis by targeting RASA1 in human umbilical vein endothelial cells. Mol Med Rep 19: 3393-3402, 2019.

13. Zhu Y, Zhao H, Rao M and Xu S: MicroRNA-365 inhibits proliferation, migration and invasion of glioma by targeting PIK3R3. Oncol Rep 37: 2185-2192, 2017.

14. Wang H, Tang C, Na M, Ma W, Jiang Z, Gu Y, Ma G, Ge H, Shen $\mathrm{H}$ and Lin Z: miR-422a inhibits glioma proliferation and invasion by targeting IGF1 and IGF1R. Oncol Res 25: 187-194, 2017.

15. Zhi T, Jiang K, Xu X, Yu T, Wu W, Nie E, Zhou X, Jin X, Zhang J, Wang Y and Liu N: MicroRNA-520d-5p inhibits human glioma cell proliferation and induces cell cycle arrest by directly targeting PTTG1. Am J Transl Res 9: 4872-4887, 2017.

16. Gu G, Wang L,Zhang J, Wang H, Tan T and Zhang G: MicroRNA-384 inhibits proliferation migration and invasion of glioma by targeting at CDC42. Onco Targets Ther 11: 4075-4085, 2018.

17. Ma J, Yu J, Liu J, Yang X, Lou M, Liu J, Feng F, Ji P and Wang L: MicroRNA-302a targets GAB2 to suppress cell proliferation, migration and invasion of glioma. Oncol Rep 37: 1159-1167, 2017.

18. Romero-Cordoba SL, Rodriguez-Cuevas S, Bautista-Pina V, Maffuz-Aziz A, D'Ippolito E, Cosentino G, Baroni S, Iorio MV and Hidalgo-Miranda A: Loss of function of miR-342-3p results in MCT1 over-expression and contributes to oncogenic metabolic reprogramming in triple negative breast cancer. Sci Rep 8: 12252, 2018.

19. Hu K, Mu X, Kolibaba H, Yin Q, Liu C, Liang X and Lu J: Metadherin is an apoptotic modulator in prostate cancer through miR-342-3p regulation. Saudi J Biol Sci 25: 975-981, 2018.

20. Zhu X, Li W, Zhang R and Liu Y: MicroRNA-342 inhibits cell proliferation and invasion in nasopharyngeal carcinoma by directly targeting ZEB1. Oncol Lett 16: 1298-1304, 2018.

21. Gentry PR, Sexton PM and Christopoulos A: Novel allosteric modulators of G protein-coupled receptors. J Biol Chem 290: 19478-19488, 2015.

22. Scholz N, Gehring J, Guan C, Ljaschenko D, Fischer R, Lakshmanan V, Kittel RJ and Langenhan T: The adhesion GPCR latrophilin/CIRL shapes mechanosensation. Cell Rep 11: 866-874, 2015.

23. Ferré S, Casadó V, Devi LA, Filizola M, Jockers R, Lohse MJ, Milligan G, Pin JP and Guitart X: G protein-coupled receptor oligomerization revisited: functional and pharmacological perspectives. Pharmacol Rev 66: 413-434, 2014.

24. Kumari P, Ghosh E and Shukla AK: Emerging approaches to GPCR ligand screening for drug discovery. Trends Mol Med 21: 687-701, 2015.

25. Kadara H, Fujimoto J, Men T, Ye X, Lotan D, Lee JS and Lotan R: A Gprc5a tumor suppressor loss of expression signature is conserved, prevalent, and associated with survival in human lung adenocarcinomas. Neoplasia 12: 499-505, 2010.

26. Liu S, Ye D, Wang T, Guo W, Song H, Liao Y, Xu D, Zhu H, Zhang Z and Deng J: Repression of GPRC5A is associated with activated STAT3, which contributes to tumor progression of head and neck squamous cell carcinoma. Cancer Cell Int 17: 34, 2017.

27. Zhou $\mathrm{H}$ and Rigoutsos I: The emerging roles of GPRC5A in diseases. Oncoscience 1: 765-776, 2014.

28. Zhou H and Rigoutsos I: MiR-103a-3p targets the 5'UTR of GPRC5A in pancreatic cells. RNA 20: 1431-1439, 2014.

This work is licensed under a Creative Commons Attribution-NonCommercial-NoDerivatives 4.0 International (CC BY-NC-ND 4.0) License. 